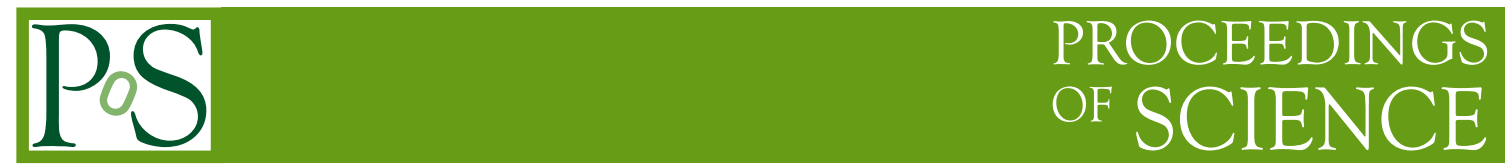

\title{
Diffractive Vector Meson Production at HERA
}

\author{
Armen Buniatyan* \\ Physics Institute of University of Heidelberg \\ E-mail: Armen. Buniatyan@desy. de \\ On behalf of the H1 and ZEUS Collaborations
}

The recent results on diffractive photoproduction of $J / \psi$ and $\Upsilon(1 S)$ mesons and on exclusive electroproduction of two pions from $\mathrm{H} 1$ and ZEUS Experiments at HERA are discussed. The measurements are used for phenomenological fits and for comparison with other measurements.

International Conference on the Structure and the Interactions of the Photon including the 20th International Workshop on Photon-Photon Collisions and the International Workshop on High Energy Photon Linear Colliders

20 - 24 May 2013

Paris, France

* Speaker. 


\section{Introduction}

Diffractive production of vector mesons (VM) and real photons $\gamma^{*} p \rightarrow V+Y$ (with $V=\rho^{0}, \omega$, $\phi, J / \psi, \Psi^{\prime}, \Upsilon$ and $\gamma$ ), in which the proton may stay intact $(Y \equiv p)$ or dissociate into a higher mass state $Y$ has been extensively studied at HERA. These processes span the whole range from "soft" diffraction, described by Regge phenomenology, to "hard" diffraction, where the hard scales, such as the heavy VM mass $\left(M_{V}\right)$ or the squared momentum transfer exchanges $Q^{2}, t$ at the respective photon and proton vertices (illustrated in Fig. 1) are involved and where the pQCD calculations can be applied. The VM production can be described in $\mathrm{PQCD}$ through the factorisation of virtual photon fluctuation into a $q \bar{q}$ colour dipole, diffractive dipole-proton scattering and $q \bar{q}$ recombination into the final state $\mathrm{VM}$ or photon. The interaction scale $\mu$ is given by the characteristic transverse size of the dipole, with $\mu^{2} \sim\left(Q^{2}+M_{V}^{2}\right)$. The dipole-proton scattering is modelled in $\mathrm{pQCD}$ as the exchange of a colour singlet two-gluon system (represented by a two-gluon exchange diagram in right side of Figure 1) or a BFKL ladder. Cross sections are thus given by the square of the gluon density in the proton.
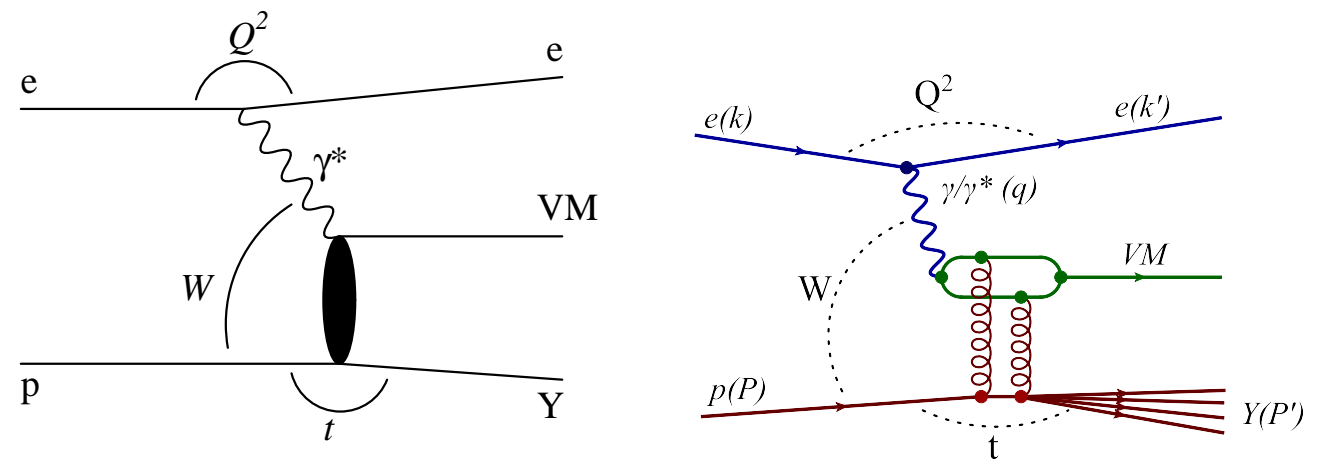

Figure 1: (Left)a generic diagram of diffractive vector meson electroproduction. (Right) a diagram of diffractive vector meson ptoduction which proceeds via the exchange of two-gluon system.

Among the most striking expectations in the transition from the soft to hard interactions is the change of the logarithmic derivative $\delta$ of the cross section with respect to the $\gamma^{*} p$ centre-of-mass energy $W$, from a value of about 0.2 in the soft regime to about 0.8 in the hard one (see Figure 2) and the decrease of the exponential slope $b$ of the differential cross section with respect to $t$, from a value of about $10 \mathrm{GeV}^{2}$ to an asymptotic value of about $5 \mathrm{GeV}^{2}$ when $\mu$ scale increases.

Experimentally the events originated from the exclusive and diffractive vector mesons production are selected using a clean experimental signatures:

- the scattered electron is either detected in the electromagnetic calorimeter or is lost in the beampipe. In the latest case the photon virtuality $Q^{2}$ is small, and these interactions are referred to as photoproduction;

- the proton either scatters elastically at very small angles and goes inside the beampipe without any energy deposit in the central detector, or it dissociates and lefts some energy in the forward part of the detector;

- the final state contains only two leptons from the VM decay. 


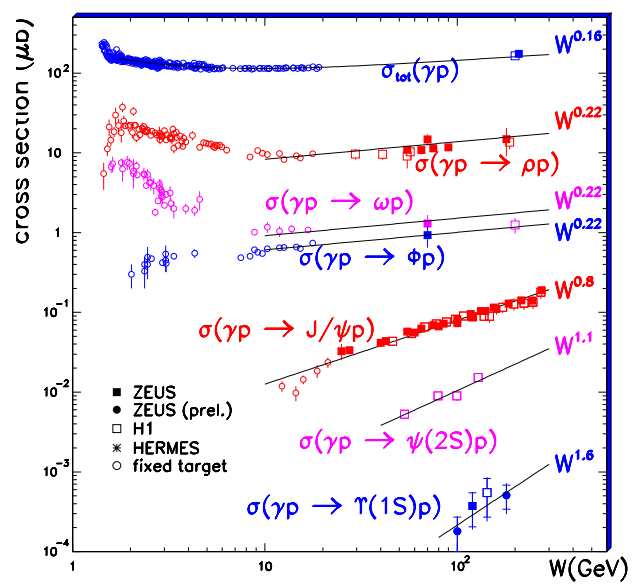

Figure 2: (left) The $W$ dependence of the cross section for diffractive VM production in the photoproduction $\left(Q^{2} \sim 0\right)$, together with fits of the form $\sigma \sim W^{\delta}$.

In this report a short review of the recent results of $\mathrm{H} 1$ and ZEUS experiments of the measurements of exclusive or diffractive production of $J / \psi$ [1], $\Upsilon(1 S)$ [2] and di-pions [3] is presented. Figure 3 shows the invariant mass distributions of di-leptons $\left(e^{+} e^{-}, \mu^{+} \mu^{-}\right)$used to reconstruct $J / \psi$ and $\Upsilon(1 S)$ discussed in this report.
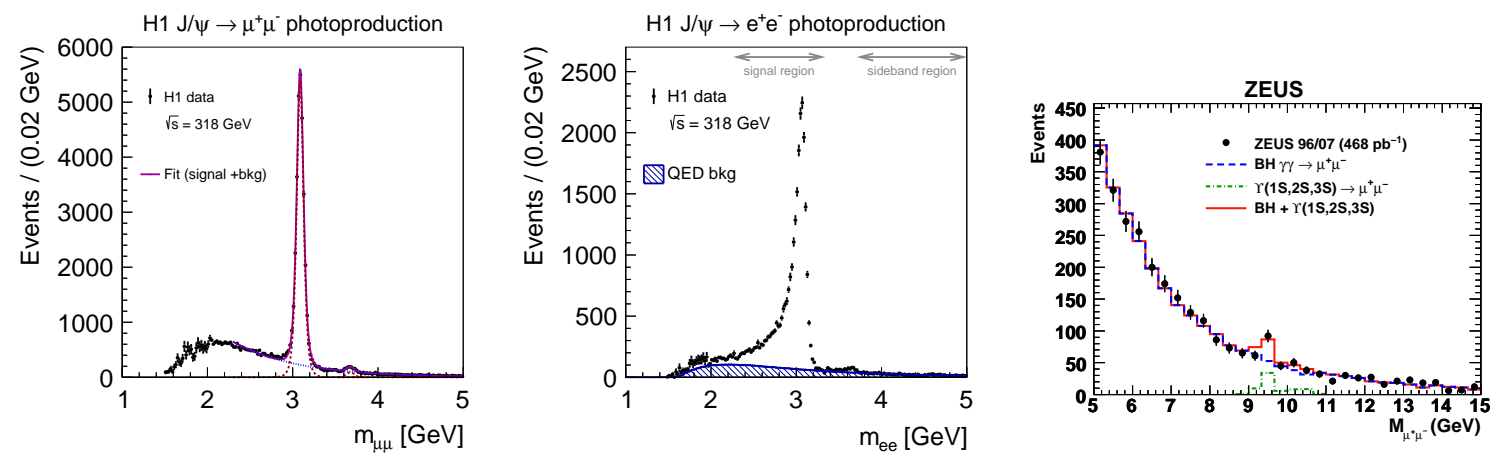

Figure 3: The dilepton invariant mass distributions in the $J / \psi \rightarrow \mu^{+} \mu^{-}, J / \psi \rightarrow e^{+} e^{-}$and $\Upsilon \rightarrow \mu^{+} \mu^{-}$ decay channels.

\section{Energy dependence of the diffractive $J / \psi$ production}

The H1 Collaboration performed a simultaneous measurement of $J / \psi$ photoproduction in elastic and proton dissociation processes [1]. The measurement results are based on two data sets: the data taken in the years 2006 - 2007, when HERA was operated with proton beam energy $920 \mathrm{GeV}$, resulting in $\sqrt{s} \approx 318 \mathrm{GeV}$, corresponding to an integrated luminosity of $130 \mathrm{pb}^{-1}$ and the data recorded in 2007 before the final HERA shutdown, when the proton beam energy was reduced to $460 \mathrm{GeV}$, resulting in $\sqrt{s}=225 \mathrm{GeV}$, corresponding to an integrated luminosity of $10.8 \mathrm{pb}^{-1}$. The $J / \psi$ mesons are reconstructed in the $J / \psi \rightarrow \mu^{+} \mu^{-}$and $e^{+} e^{-}$decay channels. Regularised unfolding is used to determine the elastic and proton-dissociative cross section in bins of $t$ and $W_{\gamma p}$.

The measured elastic and proton-dissociative cross sections as a function of $W_{\gamma p}$ are shown in Figure 4. The elastic and proton-dissociative cross sections are of similar size at the lowest $W_{\gamma p}=30 \mathrm{GeV}$ accessed in this analysis. The elastic cross section rises faster with increasing 
$W_{\gamma p}$ than the proton-dissociative one. Also shown is the ratio of the proton-dissociative to the elastic cross section as a function of $W_{\gamma p}$. The ratio decreases from 1 to 0.8 as $W_{\gamma p}$ increases from $30 \mathrm{GeV}$ to $100 \mathrm{GeV}$. The elastic and the proton-dissociative cross sections as a function of $W_{\gamma p}$ are fitted simultaneously, taking into account the correlations between these cross sections. As parametrisation a power law function of the form $\sigma=N\left(W_{\gamma p} / W_{\gamma p, 0}\right)^{\delta}$ with $W_{\gamma p, 0}=90 \mathrm{GeV}$ is used, with separate sets of parameters for the elastic and the proton-dissociative cases. The result of the fit is compared to the measurements. The fit results to the values $\delta^{e l}=0.67 \pm 0.03$ and $\delta^{p d}=0.42 \pm 0.05$.
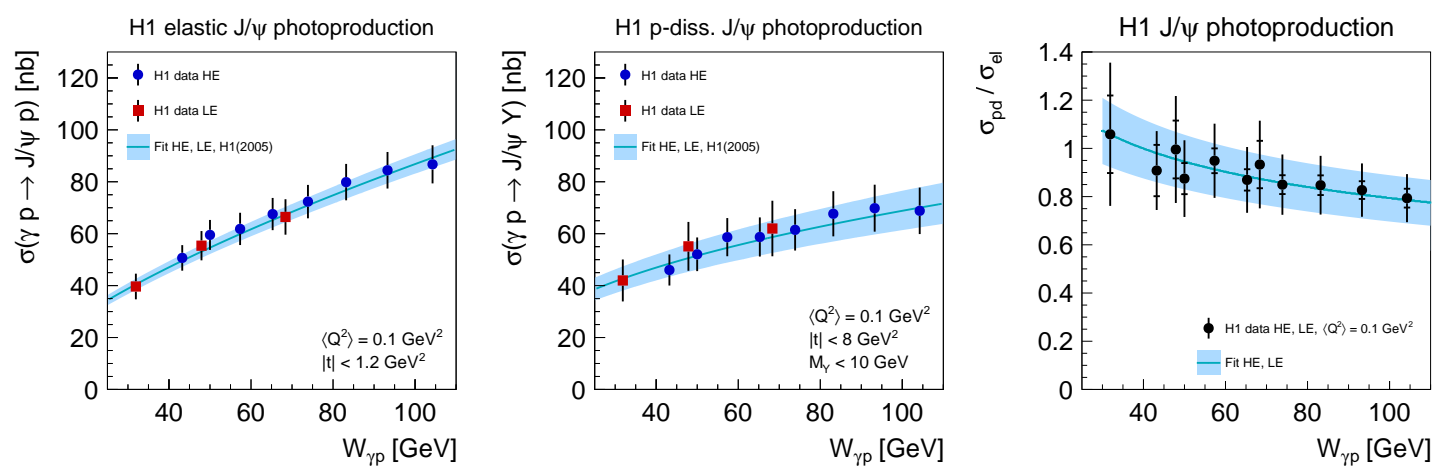

Figure 4: $J / \psi$ photoproduction cross section as function of photon proton centre-of-mass energy $W_{\gamma p}$ for the elastic and proton-dissociative regime. The rightmost figure shows the ratio of the elastic to the protondissociative $J / \psi$ photoproduction cross section as a function of $W_{\gamma p}$.

A compilation of cross section measurements for the elastic $J / \psi$ photoproduction cross section is shown in Figure 5 as a function of $W_{\gamma p}$. The data from the present analysis fall in the gap between the data of fixed target experiments [6,7] at low $W_{\gamma p}$ and the bulk of the previous high $W_{\gamma p}$ HERA data. The fixed target data exhibit a lower normalisation and a steeper slope than observed at HERA. Also shown are recent results from the LHCb experiment [8]. The extrapolated fit function for the $\mathrm{H} 1$ elastic $J / \psi$ photoproduction cross section describes the LHCb data points at high $W_{\gamma p}$ well.

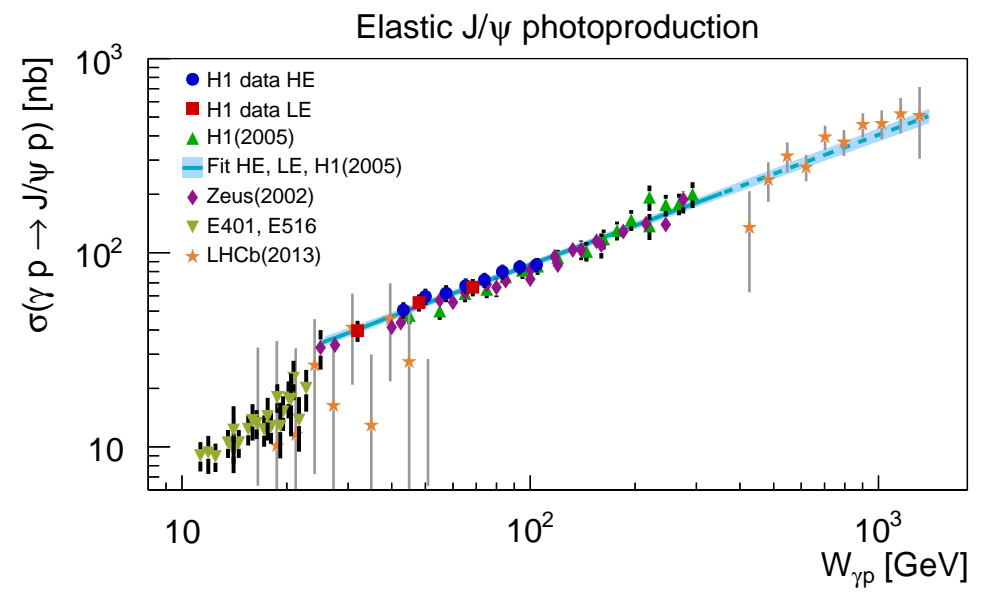

Figure 5: Compilation of various elastic $J / \psi$ photoproduction cross section measurements including this measurement, previous HERA results [4, 5], results from fixed target experiments $[6,7]$ and from the $\mathrm{LHCb}$ [8] experiment. 


\section{3. $t$ dependence of the $J / \psi$ and $\Upsilon$ photoproduction cross sections}

The measured elastic and proton-dissociative $J / \psi$ photoproduction cross sections differential in $|t|$ are shown in Figure 6 [1]. The cross sections fall steeply as a function of $|t|$, with a clear difference between the shapes of the proton-dissociative and elastic distributions. The elastic and protondissociative differential cross sections $d \sigma / d t$ are fitted simultaneously. The elastic cross section is parametrised as $d \sigma / d t=N_{e l} e^{-b_{e l}|t|}$. For the proton-dissociative cross section the parametrisation $d \sigma / d t=N_{p d}\left(1+\left(b_{p d} / n\right)|t|\right)^{-n}$ is chosen, which interpolates between an exponential at low $|t|$ and a power law behaviour at high values of $|t|$. The obtained parameterisations for the elastic and proton-dissociative cross sections are compared to the data in Figure 6. The elastic cross section data for $|t|>0.1 \mathrm{GeV}$ are well described by the exponential parametrisation. The fall of elastic cross section with increasing $|t|$ is much faster than the proton-dissociative cross section, which is reflected in the values for $b_{e l}$ and $b_{p d}: b_{e l}=(4.88 \pm 0.15) \mathrm{GeV}^{-2}$ and $b_{p d}=(1.79 \pm 0.12) \mathrm{GeV}^{-2}$.
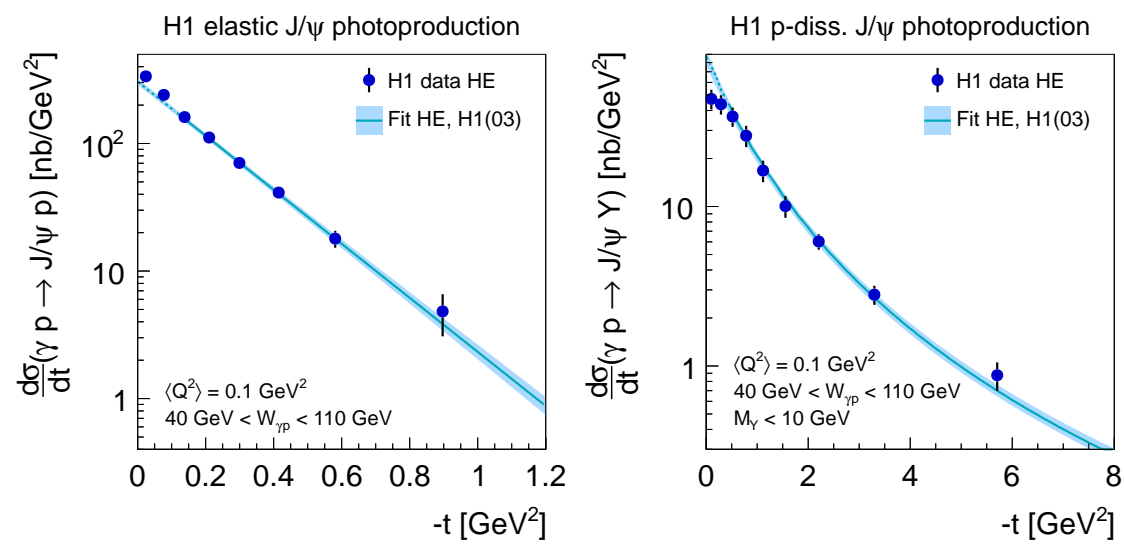

Figure 6: Differential $J / \psi$ photoproduction cross section $d \sigma / d t$ as function of the negative squared fourmomentum transfer at the proton vertex $-t$, as obtained for elastic and proton-dissociative regimes.

The ZEUS Collaboration studied the $t$ dependence of exclusive photoproduction of $\Upsilon(1 S)$ in the kinematic range $60<W<220 \mathrm{GeV}$ [2] using the data collected inthe period 1996-2007 and corresponding to an integrated luminosity of $468 \mathrm{pb}^{-1}$. The measured $t$ distribution is shown in the left side of Figure 7. The fit of $t$ distribution with a form $\exp (-b|t|)$ yields value $b=$ $4.3_{-1.3}^{+2.0}(\text { stat. })_{-0.6}^{+0.5}$ (sys. $) \mathrm{GeV}^{-2}$.

A comparison of all HERA measurements of the slope parameter $b$ for exclusive light and heavy vector meson production and for deeply inelastic Compton scattering (DVCS) is shown in the right side of Figure 7. In an optical model approach the exponential slope $b$ of the $t$-dependence of the exclusive VM production is related to the sum of squared radii of the $\gamma \rightarrow q \bar{q}$ dipole and that of the proton, and thus is a measure of transverse size of interaction region. At high values of the VM mass $M_{V M}$ or photon virtuality $Q^{2}$ the $q \bar{q}$ contribution decreases as $b_{q \bar{q}} \propto 1 /\left(Q^{2}+M_{V M}^{2}\right)$ and the slope of the $t$-dependence saturates at $b \sim 5 \mathrm{GeV}^{-2}$, which corresponds to the gluonic radii of the proton. The values of $b$ slopes measured for photoproduction of $J / \psi$ and $\Upsilon(1 S)$ are consistent with this asymptotic behaviour, as is demonstrated in the right side of Figure 7. 

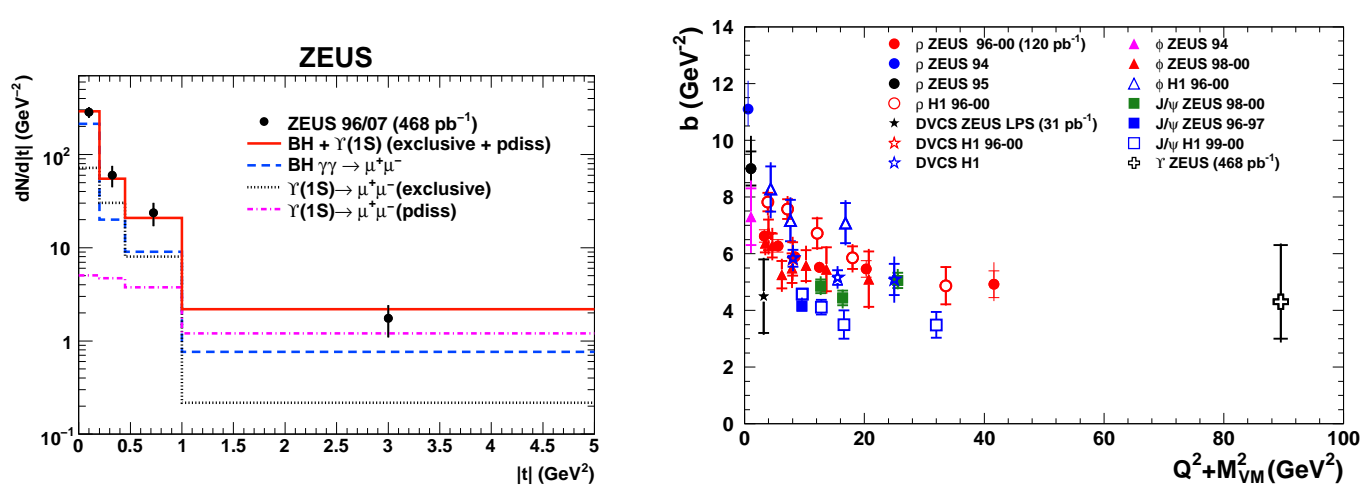

Figure 7: (Left) Measured $|t|$ distribution with error bars denoting statistical uncertainties. Fitted distributions for simulated events are shown for the Bethe-Heitler, exclusive $\Upsilon(1 S)$ and proton dissociative $\Upsilon(1 S)$ processes. The solid line shows the sum of all contributions. (Right) Comparison of the HERA measurements of the slope parameter $b$ as a function of the scale $Q^{2}+M_{V M}^{2}$ for exclusive VM production, including the exclusive $\Upsilon(1 S)$ production (the rightmost data point), and for deeply virtual Compton scattering (DVCS).

\section{Exclusive Electroproduction of two pions $e p \rightarrow \pi^{+} \pi^{-} p$}

For the light vector mesons, e.g. for $\rho$ meson, the exclusive production can be described in terms of pQCD if the hard scale is provided by the photon virtuality $Q^{2}$. Furthermore, by varying $Q^{2}$ the sensitivity to the VM wave-function can be obtained by scanning it at different $q \bar{q}$ distance scales. The excited VM states can be used to study the effect caused by changing the scanning size.

The exclusive electroproduction of two pions $\gamma^{*} p \rightarrow \pi^{+} \pi^{-} p$ in the mass range $0.4<M_{\pi \pi}<2.5 \mathrm{GeV}$ has been studied with the ZEUS detector at HERA using an integrated luminosity of $82 \mathrm{pb}^{-1}$ [3].

The $\pi^{+} \pi^{-}$mass distribution is shown in Figure 8. A clear peak is seen in the $\rho$ mass range. A small shoulder is apparent around $1.3 \mathrm{GeV}$ and a secondary peak at about $1.8 \mathrm{GeV}$. The $M_{\pi \pi}$ system consists of a resonance part, which is described by the pion form factor, and a non-resonant background. The two-pion invariant-mass distribution is related to the pion electromagnetic form factor, $F_{\pi}\left(M_{\pi \pi}\right)$, through the following relation $[9,10]$ :

$$
\frac{d N\left(M_{\pi \pi}\right)}{d M_{\pi \pi}} \propto\left[\left|F_{\pi}\left(M_{\pi \pi}\right)\right|^{2}+B\left(\frac{M_{0}}{M_{\pi \pi}}\right)^{n}\right] .
$$

The second term is a parameterization of the non-resonant background, with constant parameters $B, n$ and $M_{0}=1 \mathrm{GeV}$. There are several parameterizations of the pion form factor usually used for fitting the $\pi^{+} \pi^{-}$mass distribution. Here results based on Kuhn-Santamaria (KS) [11] parameterisation are presented. In the mass range $M_{\pi \pi}<2.5 \mathrm{GeV}$, the KS parameterization of the pion form factor includes contributions from the $\rho(770), \rho^{\prime}(1450)$ and $\rho^{\prime \prime}(1700)$ resonances,

$$
F_{\pi}\left(M_{\pi \pi}\right)=\frac{B W_{\rho}\left(M_{\pi \pi}\right)+\beta B W_{\rho^{\prime}}\left(M_{\pi \pi}\right)+\gamma B W_{\rho^{\prime \prime}}\left(M_{\pi \pi}\right)}{1+\beta+\gamma} .
$$


Here $\beta$ and $\gamma$ are relative amplitudes and $B W_{V}$ is the Breit-Wigner distribution which has the form

$$
B W_{V}\left(M_{\pi \pi}\right)=\frac{M_{V}^{2}}{M_{V}^{2}-M_{\pi \pi}^{2}-i M_{V} \Gamma_{V}\left(M_{\pi \pi}\right)},
$$

where $M_{V}$ and $\Gamma_{V}\left(M_{\pi \pi}\right)$ are the VM mass and momentum-dependent width, respectively.

The results of the fit of two-pion invariant-mass distribution with a function described above are listed in Table 1. The negative interference between all the resonances results in the $\rho^{\prime}$ signal appearing as a shoulder. To illustrate this better, the same data and fit are shown in the right side of Figure 8 on a linear scale and limited to $M_{\pi \pi}>1.2 \mathrm{GeV}$, with separate contributions from the background, the three resonant amplitudes as well as their total interference term. The masses and widths of the $\rho$ and the $\rho^{\prime \prime}$ as well as the width of the $\rho^{\prime}$ agree with those from the Particle Data Group (PDG) [12], while there is about $100 \mathrm{MeV}$ difference between the fitted mass of the $\rho^{\prime}$ and the PDG value, which however has large uncertainty. In the mass fits it was assumed that the relative amplitudes $\beta$ and $\gamma$ are real. This assumption was tested by repeated fit allowing the amplitudes to be complex. The values of the phase-shifts obtained from the fit support the assumption of the real nature of the relative amplitudes.
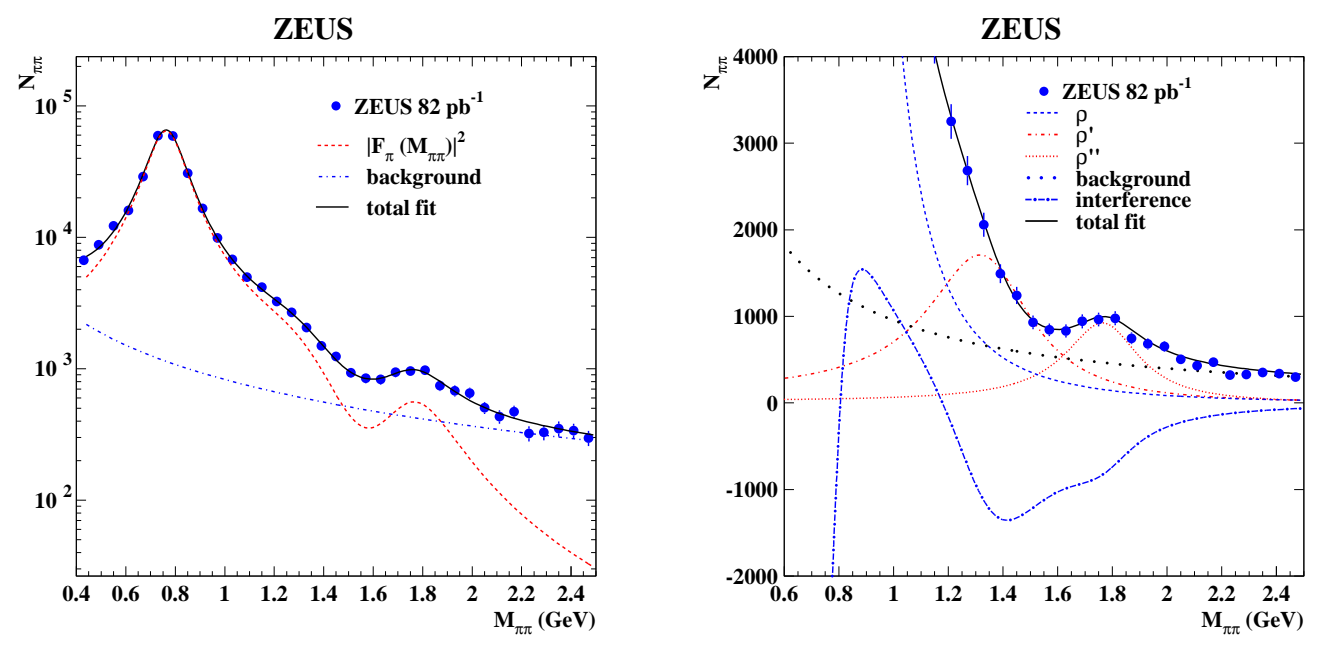

Figure 8: The two-pion invariant-mass distribution, $M_{\pi \pi}$. The dots are the data and the full line is the result of a fit using the Kuhn-Santamaria parameterization. The contributions of the three resonances $\rho, \rho^{\prime}$ and $\rho^{\prime \prime}$ and the background are shown separately.

The $Q^{2}$ dependence of the relative amplitudes was determined by performing the fit to $M_{\pi \pi}$ in three $Q^{2}$ regions within the range $2<Q^{2}<80 \mathrm{GeV}^{2}$. The masses and widths of the three resonances were fixed to the values found in the overall fit. A reasonable description of the data is achieved in all three $Q^{2}$ regions. The corresponding values of $\beta$ and $\gamma$ are given in Table 2. The absolute value of $\beta$ increases with $Q^{2}$ while the value of $\gamma$ is consistent with no $Q^{2}$ dependence within large uncertainties. The left side of Figure 9 shows the curves representing the pion form factor, $\left|F_{\pi}\left(M_{\pi \pi}\right)\right|^{2}$, as obtained in three $Q^{2}$ ranges. Also shown are results obtained in the time-like regime from the reaction $e^{+} e^{-} \rightarrow \pi^{+} \pi^{-}$[13]. In general, the features of the $\left|F_{\pi}\left(M_{\pi \pi}\right)\right|^{2}$ distribution observed here are also observed in $e^{+} e^{-}$. Above the $\rho$ region, where the interference between the 


\begin{tabular}{|l|c|c|}
\hline Parameter & ZEUS & PDG \\
\hline $\mathrm{M}_{\rho}(\mathrm{MeV})$ & $771 \pm 2_{-1}^{+2}$ & $775.49 \pm 0.34$ \\
\hline$\Gamma_{\rho}(\mathrm{MeV})$ & $155 \pm 5 \pm 2$ & $149.1 \pm 0.8$ \\
\hline$\beta$ & $-0.27 \pm 0.02 \pm 0.02$ & \\
\hline $\mathrm{M}_{\rho^{\prime}}(\mathrm{MeV})$ & $1350 \pm 20_{-30}^{+20}$ & $1465 \pm 25$ \\
\hline$\Gamma_{\rho^{\prime}}(\mathrm{MeV})$ & $460 \pm 30_{-45}^{+40}$ & $400 \pm 60$ \\
\hline$\gamma$ & $0.10 \pm 0.02_{-0.01}^{+0.02}$ & \\
\hline $\mathrm{M}_{\rho^{\prime \prime}}(\mathrm{MeV})$ & $1780 \pm 20_{-20}^{+15}$ & $1720 \pm 20$ \\
\hline$\Gamma_{\rho^{\prime \prime}}(\mathrm{MeV})$ & $310 \pm 30_{-35}^{+25}$ & $250 \pm 100$ \\
\hline$B$ & $0.41 \pm 0.03 \pm 0.07$ & \\
\hline$n$ & $1.30 \pm 0.06_{-0.13}^{+0.18}$ & \\
\hline
\end{tabular}

Table 1: Fit parameters obtained using $F_{\pi}\left(M_{\pi \pi}\right)$ parameterization. Masses and widths are in MeV. The first uncertainty is statistical, the second systematic. Also shown are the masses and widths from the PDG [12].

\begin{tabular}{|l|c|c|c|}
\hline$Q^{2}\left(\mathrm{GeV}^{2}\right)$ & $2-5$ & $5-10$ & $10-80$ \\
\hline$\beta$ & $-0.249 \pm 0.008_{-0.003}^{+0.005}$ & $-0.282 \pm 0.008_{-0.008}^{+0.005}$ & $-0.35 \pm 0.02 \pm 0.01$ \\
\hline$\gamma$ & $0.100 \pm 0.009 \pm 0.003$ & $0.098 \pm 0.012_{-0.003}^{+0.005}$ & $0.118 \pm 0.022_{-0.006}^{+0.008}$ \\
\hline
\end{tabular}

Table 2: The $Q^{2}$ dependence of the $\beta$ and $\gamma$ parameters.

$\rho^{\prime}$ and the $\rho^{\prime \prime}$ starts to dominate, there is a dependence of $\left|F_{\pi}\left(M_{\pi \pi}\right)\right|^{2}$ on $Q^{2}$, with the results from the lowest $Q^{2}$ range closest to those from $e^{+} e^{-}$. However, in the region of the $\rho$ peak the pion form-factor $\left|F_{\pi}\left(M_{\pi \pi}\right)\right|^{2}$ is highest at the highest $Q^{2}$, while the $e^{+} e^{-}$data are higher than those in the highest $Q^{2}$ range. The $e p$ and $e^{+} e^{-}$data agree within errors for $M_{\pi \pi}>1.8 \mathrm{GeV}$.

The cross section ratios of $\rho^{\prime}$ to $\rho$ and $\rho^{\prime \prime}$ to $\rho$ are investigated as a function of $Q^{2}$. The ratio $R_{V}$ defined as $R_{V}=[\sigma(V) \cdot B r(V \rightarrow \pi \pi)] / \sigma(\rho)$, where $\sigma$ is the cross section for VM production and $\operatorname{Br}(V \rightarrow \pi \pi)$ is the branching ratio of the vector meson $V\left(\rho^{\prime}, \rho^{\prime \prime}\right)$ into $\pi \pi$. The right side of Figure 9 shows the ratio $R_{V}$ for $V=\rho^{\prime}, \rho^{\prime \prime}$, as a function of $Q^{2}$. Owing to the large uncertainties of $R_{\rho^{\prime \prime}}$, no conclusion on its $Q^{2}$ behaviour can be deduced, whereas $R_{\rho^{\prime}}$ clearly increases with $Q^{2}$.

\section{Conclusions}

Presented here is a subjective selection taken from the wealth of new measurements of exclusive vector meson production processes delivered by $\mathrm{H} 1$ and ZEUS experiments at HERA collider. Much progress has been made over recent years, in the type of studies that can be performed, the precision achieved and in theoretical understanding. The high precision of the measurements can be used to refine the models and contribute to a better understanding of the vector meson wave functions and of the gluon density in the proton.

Acknowledgments I would like to thank the organizers for creating a pleasant and inspiring conference atmosphere. 

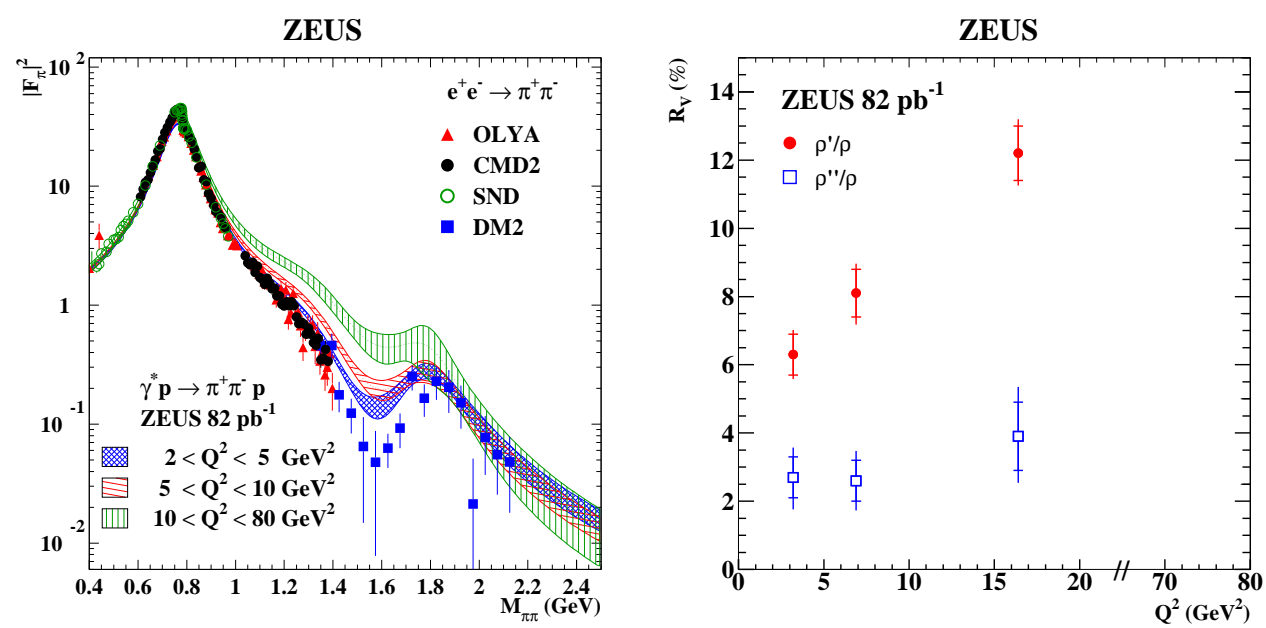

Figure 9: Left: The pion form factor squared, $\left|F_{\pi}\right|^{2}$, as a function of the $\pi^{+} \pi^{-}$invariant mass, $M_{\pi \pi}$, as obtained from the reaction $e^{+} e^{-} \rightarrow \pi^{+} \pi^{-}$[13]. The shaded bands represent the square of the pion form factor and its total uncertainty obtained in the present analysis for three ranges of $Q^{2}$.

Right: The ratio $R_{V}$ as a function of $Q^{2}$ for $V=\rho^{\prime}$ and $\rho^{\prime \prime}$. The inner error bars indicate the statistical uncertainty, the outer error bars represent the statistical and systematic uncertainty added in quadrature.

\section{References}

[1] H1 Collaboration, C. Alexa et al., Eur. Phys. J. C 73, 2466 (2013) [arxiv:1304.5162].

[2] ZEUS Collaboration, H. Abramowicz et al., Phys. Lett. B 708, 14 (2012) [arxiv:1111.2133].

[3] ZEUS Collaboration, H. Abramowicz et al., Eur. Phys. J. C 72, 1869 (2012) [arxiv:1111.4905].

[4] H1 Collaboration, A. Aktas et al., Eur. Phys. J. C 46, 585 (2006) [hep-ex/0510016].

[5] ZEUS Collaboration, S. Chekanov et al., Eur. Phys. J. C 24, 345 (2002) [hep-ex/0201043].

[6] M. E. Binkley et al., Phys. Rev. Lett. 48, 73 (1982).

[7] B. H. Denby et al., Phys. Rev. Lett. 52, 795 (1984 ).

[8] LHCb Collaboration, R. Aaij et al., J. Phys. G 40 (2013) 045001 [arXiv:1301.7084].

[9] M.V. Polyakov, Nucl. Phys. B 555, 231 (1999).

[10] B. Clerbaux and M.V. Polyakov, Nucl. Phys. A 79, 185 (2000).

[11] J.H. Kuhn and A. Santamaria, Z. Phys. C 48, 445 (1990).

[12] K. Nakamura et al. (Particle Data Group), J. Phys. G 37 (2010) 075021.

[13] L.M. Barkov et al., Nucl. Phys. B 256, 365 (1985);

DM2 Collaboration, D. Bisello et al., Phys. Lett. B 220, 312 (1989);

CMD2 Collaboration, R.R. Akhmetshin et al., Phys. Lett. B 527, 161 (2002);

CMD2 Collaboration, V.M. Aul'chenko et al., JETP Lett., 82 (2005) 743;

SND Collaboration, M.N. Achasov et al., J. Exp. Theor. Phys. 101 (2005) 1053. 